\title{
Adherence to Car Seat Tolerance Screening Differs by Indication and Patient Characteristics
}

\author{
Skyler McLaurin-Jiang ${ }^{1,2,5}$ [D $\cdot$ Morris Weinberger ${ }^{3} \cdot$ Victor Ritter $^{4} \cdot$ T. Michael O'Shea $^{1} \cdot$ Kori B. Flower $^{1}$
}

Accepted: 7 August 2021 / Published online: 17 August 2021

( ) The Author(s), under exclusive licence to Springer Science+Business Media, LLC, part of Springer Nature 2021

\begin{abstract}
Objectives To assess whether adherence to institutional car seat tolerance screening (CSTS) guidelines differed for infants born preterm (PTM), term low birth weight (T-LBW), or both preterm and low birth weight (P-LBW), and to examine the association between CSTS adherence and patient characteristics.

Study Design Within two large academic and community hospitals, we retrospectively reviewed all infants meeting institutional criteria ( $<37$ weeks' gestation and/or $<2.27 \mathrm{~kg}$ ) for CSTS from 2014 to 2018. Multivariable logistic regression evaluated the association of patient characteristics with institutional CSTS guideline adherence.

Results 4374 eligible infants were born PTM (50.9\%), T-LBW (6.5\%), or P-LBW (42.6\%). Adherence rates were 92.7\% in the neonatal intensive care unit (NICU) and $95.2 \%$ in the well-baby nursery with initial CSTS failure rates of $6.1 \%$ and $9.9 \%$, respectively. Adherence was lowest among T-LBW (80.7\%) compared to PTM (95.1\%) or P-LBW (92.2\%) infants in the NICU ( $p<0.001)$ and well-baby nursery $(81.6 \%, 96.7 \%$ and $97.1 \%$, respectively, $\mathrm{p}<0.001)$. In bivariate analyses, gestational age, birth weight, insurance, race, hospital type, discharge year, and preferred language were associated with adherence. In fully-adjusted models, adherence was positively associated with lower gestational age, higher birth weight, non-Medicaid insurance, and later discharge year (NICU) and lower gestational age and later discharge year (well-baby nursery).

Conclusions Adherence was lower for T-LBW than PTM or P-LBW infants, despite similar CSTS failure rates. Disparities in adherence among Medicaid-insured patients in the NICU warrant further study. Future studies are needed to clarify the benefit of CSTS and increase adherence in high-risk populations.
\end{abstract}

Keywords Adherence $\cdot$ Car seat tolerance screen $\cdot$ Late preterm $\cdot$ Low birth weight $\cdot$ Disparities

Skyler McLaurin-Jiang

Skyler.mclaurin@ttuhsc.edu

1 Department of Pediatrics, University of North Carolina at Chapel Hill School of Medicine, Chapel Hill, NC, USA

2 Cecil G. Sheps Center for Health Services Research, University of North Carolina at Chapel Hill, Chapel Hill, NC, USA

3 Department of Health Policy and Management, Gillings School of Global Public Health, University of North Carolina-Chapel Hill, Chapel Hill, NC, USA

4 Department of Biostatics, Gillings School of Global Public Health, University of North Carolina-Chapel Hill, Chapel Hill, NC, USA

5 Department of Pediatrics, Texas Tech University Health Sciences Center School of Medicine, 1400 Wallace Blvd, Amarillo, TX 79106, USA

\begin{abstract}
Abbreviations
CSTS Car seat tolerance screening

NICU Neonatal intensive care unit

LBW Low birth weight

P-LBW Preterm and low birth weight

T-LBW Term and low birth weight
\end{abstract}

\section{Significance Statement}

What is already known on this subject?

An increasing number of preterm and low birth weight newborns are undergoing car seat tolerance screening. These infants are at increased risk for cardiorespiratory compromise associated with a semi-upright position while situated in infant safety seats.

What this study adds?

We highlight the differences in CSTS adherence by indication for screening and socioeconomic status. These 
findings underscore the need for clarification of national and local CSTS guidelines and understanding of provider decision making with respect to car seat screening.

\section{Introduction}

The American Academy of Pediatrics (AAP) recommends car seat tolerance screening (CSTS) to detect episodes of apnea, bradycardia, or oxygen desaturations in premature and/or low birth weight infants prior to initial hospital discharge (Bull et al., 2009). In recent decades, CSTS has been broadly implemented in well-baby nurseries and neonatal intensive care units (NICUs) across the United States (US). Standard processes for conducting a CSTS have not been established (Williams \& Martin, 2003). Initial CSTS recommendations were based on the increased risk of hypoventilation among preterm infants while in car seats (Willett et al., 1986), but low birth weight infants have similar rates of failing CSTS (Bass, 2015; Davis, 2015; McLaurin-Jiang et al., 2019). Among the 500,000 US-born infants that are preterm and/or low birth weight each year (Martin et al., 2018), the observed rate of CSTS failure ranges from 4 to 10\% (Davis et al., 2013; Jensen et al., 2018; Schutzman et al., 2013).

Given the different settings in which preterm and low birth weight newborns receive care, CSTS adherence may vary. For instance, a 2003 survey of 72 level 1 (well-baby), 2 (special care nursery), or 3 (NICU) nurseries found that $75 \%$ of the hospitals had instituted a CSTS program (Williams \& Martin, 2003). However, adherence to the AAP recommendations varied by level of nursery with only $19 \%$ of well-baby nurseries compared to $78 \%$ of level 3 NICUs conducting CSTS screening at that time (Williams \& Martin, 2003). As rates of late preterm births increase in the US
(Huff et al., 2019), lower level nurseries may increasingly care for late preterm and low birth weight infants.

Annually $2 / 3$ of out of home sudden infant death syndrome is associated with car seat use (Liaw et al., 2019). However, whether CSTS prevents morbidity associated with car seat use among infants at-risk is unknown (Pilley $\&$ McGuire, 2006). Recently, a national survey of well-baby nurseries found wide variation in CSTS and in providers' thoughts about the utility of CSTS (Davis \& Cheo, 2020).

To our knowledge, no study has examined current rates of adherence to CSTS policies in both well-baby nursery (level 1) and NICU settings (levels 2-5). Our primary aim was to describe CSTS adherence by indication (preterm birth and/ or low birth rate). Our secondary aim was to evaluate infant characteristics associated with CSTS adherence.

\section{Methods}

We conducted a retrospective review of electronic health record (EHR) data extracted from a central data repository at one academic and one community hospital in a single health care system. Both hospitals have well-baby nurseries. The NICUs are level 5 (academic hospital) and level 3 (community hospital). We included infants born alive who met hospital criteria for CSTS and were discharged from either hospital between April 5, 2014 (when Epic ® EHR was implemented) and December 31, 2018 (Fig. 1). We excluded infants who had a discharge status other than "home" or that required tracheostomy or home ventilator as these infants may undergo non-standard CSTS given need for additional monitors or equipment.

A CSTS protocol has been in effect within this hospital system for more than 10 years and was not altered during the study period. The protocol was based on the AAP

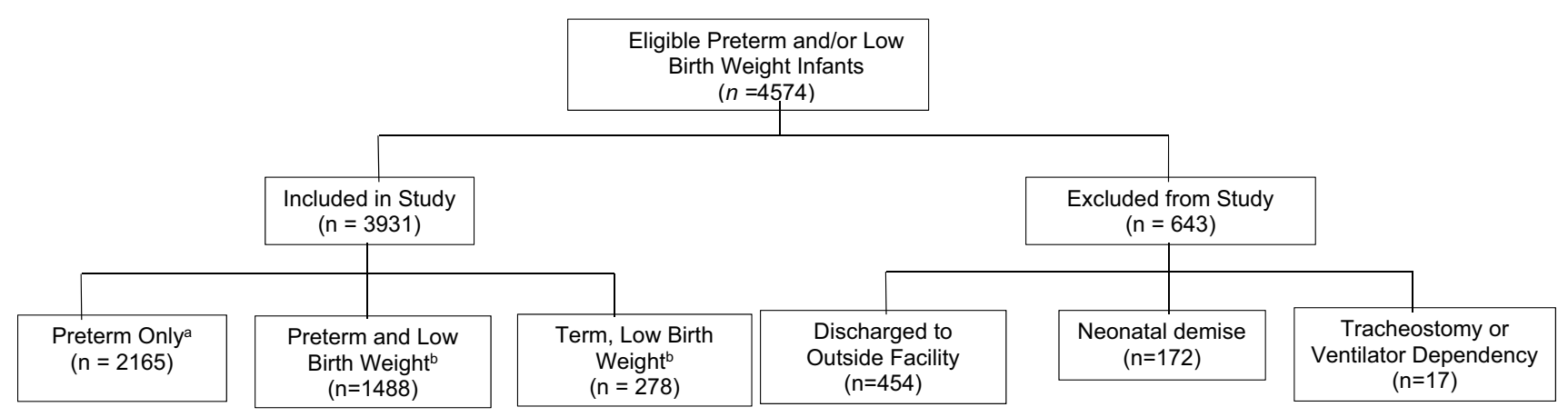

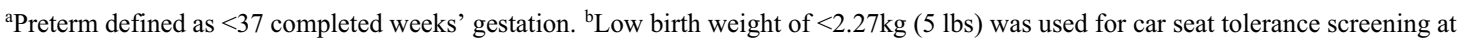
this institution.

Fig. 1 Flow diagram of study population 
Recommendations for Safe Transportation of Preterm and Low Birth Weight Newborns at Hospital Discharge (Bull et al., 2009). All infants born $<37$ weeks gestation (PTM) and/or $<2.27 \mathrm{~kg}$ (LBW) were routinely screened prior to discharge. Based on these eligibility criteria, three groups of infants were identified: (1) preterm, appropriate weight for gestational age (PTM), (2) preterm, low birth weight (P-LBW), and (3) term, low birth weight (T-LBW) infants. Although providers may choose to screen additional infants not meeting one of these criteria (e.g., term infants with Trisomy 21 or congenital heart disease), for this analysis, we focused only on infants specified in the hospital policy.

CSTS is conducted by a trained registered nurse in either the well nursery or NICU using the infant's own car seat within $24 \mathrm{~h}$ of expected hospital discharge. The infant's heart rate, respiratory rate, and oxygen saturations are measured for $90 \mathrm{~min}$ or the duration of the car ride home from the hospital (whichever is longer). A certified car seat technician with expertise in safe car seat installation is available to assist as needed with optimal infant positioning.

The primary outcome was adherence to hospital CSTS policy as defined by having met institutional criteria for screening and receiving a screen (yes/no). All CSTS results are logged by the infant's nurse in a discrete data field within the EHR. Results are documented as "pass" or "fail" and verified by the physician prior to infant's discharge.

Covariates were similarly extracted from discrete data fields in the EHR. Race/ethnicity (non-Hispanic White, nonHispanic Black, Hispanic or Latino, Asian, Other/Unknown) is typically completed by administrative staff upon maternal admission. We used maternal race/ethnicity as infant race/ ethnicity was often undocumented. Infant sex (male/female), gestational age (weeks), birth weight (kilograms), pregnancy type (singleton/multiple gestation), hospital discharge unit (NICU, well nursery, or pediatric floor), caffeine exposure, substance exposure (to alcohol, cocaine, or opiates), Apgar 1 and $5 \mathrm{~min}$, hospital type, discharge disposition (home with self-care vs. transfer to acute care facility), and discharge year were also extracted. To account for the incomplete data in 2014, we examined the trend in adherence using quarter of year as a continuous variable. There were 19 quarters over the course of this study period.

Additional maternal covariates included insurance payor (Medicaid, private, or other/unknown), maternal age, preferred language of mother (English or Spanish), mode of delivery (vaginal or cesarean), and parity. Rates of missing data were low $(<1 \%)$ for most variables other than insurance payor (8.7\%) and 1- and 5-min Apgar scores (2.2\%). Only individuals with complete data were utilized in the analyses.

We identified maternal and infant diagnoses (Supplemental Table 1) and medication exposures (caffeine) for which we hypothesized associations with providers' perception of risk of apnea or bradycardia and thus might have influenced their decision to conduct screening.

\section{Statistical Analysis}

In bivariate analyses, Chi-square or Fisher Exact tests (for categorical variables and t-tests or Wilcoxon Rank sum tests (for continuous variables) were used to determine the association of patient characteristics with CSTS adherence. In multivariable logistic regression models to examine adherence to CSTS policy, we included the following covariates chosen a priori: race/ethnicity, gestational age, birth weight, sex, Medicaid status, preferred language, hospital and discharge year. Because of significant differences in the NICU and well nursery populations, we stratified analyses by hospital unit (Supplemental Table 2). We used StataIC version 15 (Stata Corp, College Station, TX) for all statistical analyses. The University of North Carolina at Chapel Hill Institutional Review Board approved this study which was performed in accordance with the ethical standards laid down in the 1964 Declaration of Helsinki and its later amendments.

\section{Results}

Of the 4574 potentially eligible preterm and/or low birth weight infants, 643 were excluded because of discharge to an outside facility, tracheostomy and/or ventilator dependency, or not surviving to discharge (Fig. 1). The final sample comprised 3931 infants born to 3756 mothers over the 4.75 years study period.

Infants were born preterm (PTM, 55.1\%), both preterm and low birth weight (P-LBW, 34.0\%), or term and low birth weight (T-LBW, 7.1\%). Adherence rates were $92.7 \%$ in the NICU and $95.2 \%$ in the well-baby nursery with initial CSTS failure rates of $6.1 \%$ and $9.9 \%$, respectively (Table 1). Babies who were transferred to and subsequently discharged from the pediatric floor had the lowest adherence rates (Table 1). Adherence was higher among infants at the community hospital compared to the academic hospital $(96.1 \%$ vs. $91.4 \%$, $\mathrm{p}<0.001)$.

\section{NICU}

In bivariate analysis, the mean maternal age was higher among infants undergoing CSTS compared to unscreened infants $(31.2 \pm 5.9$ vs. $29.9 \pm 6.0$ years, $p=0.014)$. Adherence was lowest among T-LBW (80.7\%) compared to PTM $(95.1 \%)$ or P-LBW $(92.2 \%)$ infants, $\mathrm{p}<0.001$. Adherence varied by race/ethnicity with greater adherence among nonHispanic White and Asian mothers compared to non-Hispanic Black, Hispanic, or unknown race/ethnicity (Table 2). Males were more likely to undergo CSTS (93.9\% vs. $91.2 \%$, 
Table 1 Baseline characteristics of infants meeting institutional criteria for car seat tolerance screening (April 2014-

December 2018)

\begin{tabular}{|c|c|c|c|c|}
\hline & $\begin{array}{l}\text { Overall distribution } \\
N=3931 \text { (100.0) }\end{array}$ & $\begin{array}{l}\text { Screened } \\
N=3860(93.6)\end{array}$ & $\begin{array}{l}\text { Not screened } \\
N=251(6.4)\end{array}$ & $\mathrm{p}$-value \\
\hline Reason for inclusion & & & & $<0.001$ \\
\hline Preterm only ${ }^{\mathrm{a}}$ & $2165(55.1)$ & $2074(95.8)$ & $91(4.2)$ & \\
\hline Preterm and low birth weight ${ }^{\mathrm{b}}$ & $1488(34.0)$ & $1381(92.8)$ & $107(7.2)$ & \\
\hline Term and low birth weight & $278(7.1)$ & $225(80.9)$ & $53(19.1)$ & \\
\hline \multicolumn{5}{|l|}{ Maternal factors } \\
\hline Age (in years) & & & & 0.530 \\
\hline $15-19$ & $108(2.8)$ & $98(90.7)$ & $10(9.3)$ & \\
\hline $20-24$ & $484(12.3)$ & $446(92.2)$ & $28(7.9)$ & \\
\hline $25-29$ & $1012(25.8)$ & $950(93.9)$ & $62(6.1)$ & \\
\hline $30-34$ & $1284(32.7)$ & $1203(93.7)$ & $81(6.3)$ & \\
\hline $35-39$ & $779(19.8)$ & $733(94.1)$ & $46(5.9)$ & \\
\hline $40+$ & $262(6.7)$ & $248(94.7)$ & $14(5.3)$ & \\
\hline Parity & & & & 0.230 \\
\hline 0 & $1683(45.4)$ & $1583(94.1)$ & $100(5.9)$ & \\
\hline$\geq 1$ & $2024(54.6)$ & $1884(93.1)$ & $140(6.9)$ & \\
\hline Mode of delivery & & & & 0.226 \\
\hline Vaginal & $1971(50.3)$ & $1854(94.1)$ & $117(5.9)$ & \\
\hline Cesarean & 1947 (49.7) & $1813(93.1)$ & $134(6.9)$ & \\
\hline \multicolumn{5}{|l|}{ Pregnancy type } \\
\hline Singleton & $3412(89.9)$ & $3169(92.9)$ & $243(7.1)$ & 0.898 \\
\hline Multiple $^{c}$ & $342(9.1)$ & $317(92.7)$ & $25(7.3)$ & \\
\hline Preeclampsia & & & & 0.569 \\
\hline No preeclampsia & $1715(87.7)$ & $1591(92.8)$ & $124(7.2)$ & \\
\hline Preeclampsia & $241(12.3)$ & $226(93.8)$ & $15(6.2)$ & \\
\hline Language preference & & & & 0.171 \\
\hline English & $3574(92.8)$ & $3351(93.8)$ & $223(6.2)$ & \\
\hline Spanish & $276(7.2)$ & $253(91.7)$ & $23(8.3)$ & \\
\hline Maternal insurance type & & & & $<0.001$ \\
\hline Medicaid & $1130(30.4)$ & $1032(91.3)$ & $142(5.5)$ & \\
\hline Private/other & $2586(69.6)$ & $2444(94.5)$ & $98(8.7)$ & \\
\hline \multicolumn{5}{|l|}{ Newborn factors } \\
\hline Gestational age (weeks) & & & & $<0.001$ \\
\hline$<32$ weeks & $459(11.8)$ & $424(92.4)$ & $35(7.6)$ & \\
\hline $32-33$ weeks & $409(10.5)$ & 384(93.9) & $25(6.1)$ & \\
\hline 34-36 weeks & $2785(71.3)$ & $2647(95.0)$ & $138(5.0)$ & \\
\hline 37-38 weeks & $233(6.0)$ & $188(80.7)$ & $45(19.3)$ & \\
\hline$\geq 39$ weeks & $20(0.5)$ & $14(70.0)$ & $6(30.0)$ & \\
\hline Birth weight $(\mathrm{g})$ & & & & $<0.001$ \\
\hline$\leq 1250$ & $267(6.9)$ & $238(89.1)$ & $29(10.9)$ & \\
\hline $1251-1750$ & $351(9.0)$ & $327(93.2)$ & $24(6.8)$ & \\
\hline $1751-2250$ & $1139(29.3)$ & $1035(90.9)$ & $104(9.1)$ & \\
\hline $2251-2750$ & $1275(32.8)$ & $1231(96.6)$ & $44(3.4)$ & \\
\hline$\geq 2751$ & $856(22.0)$ & $808(94.4)$ & $48(5.6)$ & \\
\hline Sex & & & & 0.292 \\
\hline Female & $1851(47.1)$ & $1623(87.7)$ & $228(12.3)$ & \\
\hline Male & 2079 (52.9) & $1844(88.7)$ & $235(11.3)$ & \\
\hline Race/ethnicity & & & & $<0.001$ \\
\hline White, non-Hispanic & $2142(54.5)$ & $1906(89.6)$ & $236(9.4)$ & \\
\hline Black, non-Hispanic & $884(22.5)$ & $750(84.8)$ & $57(15.2)$ & \\
\hline Hispanic or Latinx & $514(13.1)$ & $453(88.2)$ & $61(11.8)$ & \\
\hline
\end{tabular}


Table 1 (continued)

\begin{tabular}{|c|c|c|c|c|}
\hline & $\begin{array}{l}\text { Overall distribution } \\
N=3931(100.0)\end{array}$ & $\begin{array}{l}\text { Screened } \\
N=3860(93.6)\end{array}$ & $\begin{array}{l}\text { Not screened } \\
N=251(6.4)\end{array}$ & p-value \\
\hline Asian or Asian American & $161(4.1)$ & $154(95.5)$ & $7(4.5)$ & \\
\hline Unknown and/or other & $227(5.8)$ & $193(84.9)$ & $35(15.1)$ & \\
\hline Discharge unit & & & & $<0.001$ \\
\hline Well nursery & $2012(51.4)$ & $1916(95.2)$ & $96(4.8)$ & \\
\hline NICU or special care nursery & $1811(46.2)$ & $1679(92.7)$ & $132(7.3)$ & \\
\hline Pediatric floor & $95(2.4)$ & $77(81.1)$ & $18(18.9)$ & \\
\hline Apnea of newborn & & & & 0.717 \\
\hline Yes & $672(17.1)$ & $627(93.3)$ & $45(6.7)$ & \\
\hline No & 3259 (82.9) & $3053(93.7)$ & $206(6.3)$ & \\
\hline Caffeine exposure & & & & $<0.001$ \\
\hline Yes & $518(13.2)$ & $484(93.4)$ & $34(6.6)$ & \\
\hline No & $3413(86.8)$ & $3196(93.6)$ & $217(6.4)$ & \\
\hline Substance exposure & & & & 0.101 \\
\hline Yes & $84(2.1)$ & $75(89.3)$ & $9(10.7)$ & \\
\hline No & 3847 (97.9) & $3605(93.7)$ & $242(6.3)$ & \\
\hline Hospital type & & & & $<0.001$ \\
\hline Academic & $2076(52.8)$ & $1898(91.4)$ & $178(8.6)$ & \\
\hline Community & $1855(47.2)$ & $1782(96.1)$ & $72(3.9)$ & \\
\hline Year & & & & $<0.001$ \\
\hline 2014 & $484(12.4)$ & $430(88.8)$ & $54(11.2)$ & \\
\hline 2015 & $868(22.2)$ & $800(92.2)$ & $68(7.8)$ & \\
\hline 2016 & 873 (22.4) & 817 (93.6) & $56(6.4)$ & \\
\hline 2017 & 846 (21.7) & 819 (96.8) & 27 (3.2) & \\
\hline 2018 & 835 (21.4) & 814 (97.5) & $21(2.5)$ & \\
\hline
\end{tabular}

Data presented are number (percent) or mean \pm standard deviation unless otherwise specified. Bold values represent significance at $\mathrm{p}<0.05$

${ }^{\text {a }}$ Preterm defined as gestational age $<37$ completed weeks

${ }^{b}$ Low birth weight criteria at this institution was $<2.27 \mathrm{~kg}$

${ }^{\mathrm{c}}$ Multiple gestation totals were based on unique maternal identification numbers such that no mother was counted more than once

$\mathrm{p}=0.28)$. The academic NICU had lower adherence than the community NICU (89.8\% vs. 97.0\%, p < 0.001). Adherence significantly increased from 2014 to 2018 (uOR 1.06, 95\% CI 1.02, 1.10, Fig. 2).

In the fully-adjusted multivariable model (Table 3), a $1 \mathrm{~kg}$ increase in birth weight was associated with 2.05 greater odds of adherence (95\% CI 1.45, 2.90). Discharge from the community hospital was associated with higher adherence (aOR 6.22, 95\% CI 4.17, 9.30) while Medicaid insurance was associated with lower adherence $(0.62,95 \%$ CI $0.48,0.80)$.

\section{Nursery}

In bivariate analysis, there was no difference in mean maternal age between infants by adherence. T-LBW infants had lower CSTS adherence rates than PTM or P-LBW infants $(81.6 \%$ vs. $96.7 \%$ and $97.1 \%$, p < 0.001$)$. Screened infants had a lower mean gestational age $(36.8 \pm 1.1$ vs.
$36.2 \pm 0.78$ weeks, $\mathrm{p}<0.001)$. Compared to infants who were $>2.25 \mathrm{~kg}$, infants $\leq 2.25 \mathrm{~kg}$ had lower rates of adherence (Table 2). Adherence significantly increased each year from 2014 to 2018 (unadjusted OR 1.06, 95\% CI 1.02, 1.10, Fig. 2).

In the fully adjusted multivariable model (Table 3), later study quarter was associated with higher adherence (aOR $1.74,95 \%$ CI 1.09, 2.79).

\section{Analysis of Academic vs. Community NICU}

In both hospitals, adherence was lower among T-LBW infants than PTM or P-LBW infants, and adherence was inversely associated with gestational age (Supplemental Table 2). At the academic hospital, adherence was lower among infants with Medicaid insurance compared to nonMedicaid insurance $(87.4 \%$ vs. $91.8 \%, p=0.020)$ and later study quarter was associated with higher adherence (unadjusted OR 1.06, 95\% 1.02, 1.11). At the community hospital, 
Table 2 Bivariate associations of infant characteristics with adherence to car seat tolerance screening in the NICU and nursery (April 2014-December 2018)

\begin{tabular}{|c|c|c|c|c|}
\hline & \multicolumn{2}{|l|}{$\begin{array}{l}\text { NICU } \\
1679(92.2)\end{array}$} & \multicolumn{2}{|c|}{$\begin{array}{l}\text { Nursery } \\
\mathrm{N}=1916(95.2)\end{array}$} \\
\hline & Screened & $p$-value & Screened & p-value \\
\hline Inclusion reason & & $<0.001$ & & $<0.001$ \\
\hline Preterm only ${ }^{\mathrm{a}}$ & $539(95.1)$ & & $1483(96.7)$ & \\
\hline Preterm and low birth weight & $1094(92.2)$ & & $264(97.1)$ & \\
\hline Term and low birth weight ${ }^{b}$ & $46(80.7)$ & & $169(81.6)$ & \\
\hline \multicolumn{5}{|l|}{ Maternal factors } \\
\hline Age (years) & & 0.242 & & 0.779 \\
\hline $15-19$ & $42(91.3)$ & & $47(94.0)$ & \\
\hline $20-24$ & $219(89.8)$ & & $214(96.0)$ & \\
\hline $25-29$ & $425(92.6)$ & & $503(96.2)$ & \\
\hline $30-34$ & $551(92.5)$ & & $629(95.0)$ & \\
\hline $35-39$ & $328(94.5)$ & & $392(94.2)$ & \\
\hline $40+$ & $114(95.8)$ & & $129(94.9)$ & \\
\hline Parity & & 0.113 & & 0.792 \\
\hline 0 & $756(93.8)$ & & $790(95.0)$ & \\
\hline$\geq 1$ & $829(91.8)$ & & $1015(95.2)$ & \\
\hline Mode of delivery & & 0.302 & & 0.717 \\
\hline Vaginal & $725(93.4)$ & & $1076(95.1)$ & \\
\hline Cesarean & $1063(92.2)$ & & $830(95.4)$ & \\
\hline Pregnancy type & & 0.773 & & 0.689 \\
\hline Singleton & $1462(92.1)$ & & $1704(94.7)$ & \\
\hline Multiple gestation ${ }^{\mathrm{c}}$ & $184(92.9)$ & & $179(95.2)$ & \\
\hline Preeclampsia & & 0.728 & & 0.297 \\
\hline No preeclampsia & $752(91.5)$ & & $779(95.1)$ & \\
\hline Preeclampsia & $115(90.6)$ & & $109(97.3)$ & \\
\hline Language preference & & 0.024 & & 0.928 \\
\hline English & $1555(93.1)$ & & $1726(95.2)$ & \\
\hline Spanish & $97(87.4)$ & & $144(95.4)$ & \\
\hline Maternal insurance & & $<0.001$ & & 0.850 \\
\hline Medicaid & $487(89.0)$ & & $508(95.0)$ & \\
\hline Private/other & $1105(94.5)$ & & $1298(95.2)$ & \\
\hline \multicolumn{5}{|l|}{ Newborn factors } \\
\hline Gestational age (weeks) & & 0.003 & & $<0.001$ \\
\hline$<32$ & $419(92.9)$ & & - & \\
\hline $32-33$ & $376(93.8)$ & & - & \\
\hline $34-36$ & $838(92.9)$ & & $1746(96.8)$ & \\
\hline $37-38$ & $34(77.3)$ & & $144(81.8)$ & \\
\hline$\geq 39$ & - & & $13(68.4)$ & \\
\hline Birth weight $(\mathrm{g})$ & & 0.068 & & $<0.001$ \\
\hline$\leq 1250$ & $235(90.0)$ & & - & \\
\hline $1251-1750$ & $320(93.0)$ & & - & \\
\hline $1751-2250$ & $582(91.5)$ & & $427(90.9)$ & \\
\hline $2251-2750$ & $366(95.3)$ & & $833(97.4)$ & \\
\hline$\geq 2751$ & $140(94.6)$ & & $649(95.4)$ & \\
\hline Sex & & 0.028 & & 0.632 \\
\hline Female & $750(91.2)$ & & $930(95.0)$ & \\
\hline Male & $929(93.9)$ & & $986(95.5)$ & \\
\hline Race/ethnicity & & 0.006 & & 0.529 \\
\hline Non-Hispanic White & $939(94.3)$ & & $1036(95.7)$ & \\
\hline Non-Hispanic Black & $384(90.1)$ & & $385(93.9)$ & \\
\hline
\end{tabular}


Table 2 (continued)

\begin{tabular}{|c|c|c|c|c|}
\hline & \multicolumn{2}{|l|}{$\begin{array}{l}\text { NICU } \\
1679(92.2)\end{array}$} & \multicolumn{2}{|c|}{$\begin{array}{l}\text { Nursery } \\
\mathrm{N}=1916(95.2)\end{array}$} \\
\hline & Screened & $p$-value & Screened & p-value \\
\hline Hispanic or Latino & $194(89.0)$ & & $261(95.3)$ & \\
\hline Asian or Asian American & $51(98.1)$ & & $109(97.3)$ & \\
\hline Unknown and/or other & $92(92.9)$ & & $110(95.3)$ & \\
\hline Apnea of newborn & & 0.148 & & \\
\hline Yes & $601(93.9)$ & & - & \\
\hline No & $1078(92.1)$ & & - & \\
\hline Caffeine exposure & & 0.236 & & \\
\hline Yes & $475(93.9)$ & & - & \\
\hline No & $1204(92.3)$ & & - & \\
\hline Substance exposure & & 0.971 & & 0.291 \\
\hline Yes & $39(92.9)$ & & $22(100.0)$ & \\
\hline No & $1640(92.7)$ & & $1897(95.2)$ & \\
\hline Hospital type & & $<0.001$ & & 0.494 \\
\hline Academic & $968(89.8)$ & & $850(94.9)$ & \\
\hline Community & $711(97.0)$ & & $1066(95.5)$ & \\
\hline Year & & 0.005 & & $<0.001$ \\
\hline 2014 & $206(89.2)$ & & $215(90.0)$ & \\
\hline 2015 & $381(93.4)$ & & $418(91.9)$ & \\
\hline 2016 & $368(93.2)$ & & $431(95.0)$ & \\
\hline 2017 & $387(95.8)$ & & $415(98.1)$ & \\
\hline 2018 & $337(96.0)$ & & $437(99.1)$ & \\
\hline
\end{tabular}

Bold values represent significance at $\mathrm{p}<0.05$

Data presented are number (percent) or mean \pm standard deviation unless otherwise specified

${ }^{\text {a }}$ Preterm defined as gestational age $<37$ completed weeks

${ }^{\mathrm{b}}$ Low birth weight criteria was $<2.27 \mathrm{~kg}$

${ }^{\mathrm{c}}$ Multiple gestation totals were based on unique maternal identification numbers such that no mother was counted more than once

- indicates cell size $<5$

Fig. 2 Adherence to car seat tolerance screening in NICU and nursery by quarter, 2014-2018

\section{Adherence to Car Seat Tolerance Screening in NICU and Nursery} by Quarter, 2014-2018

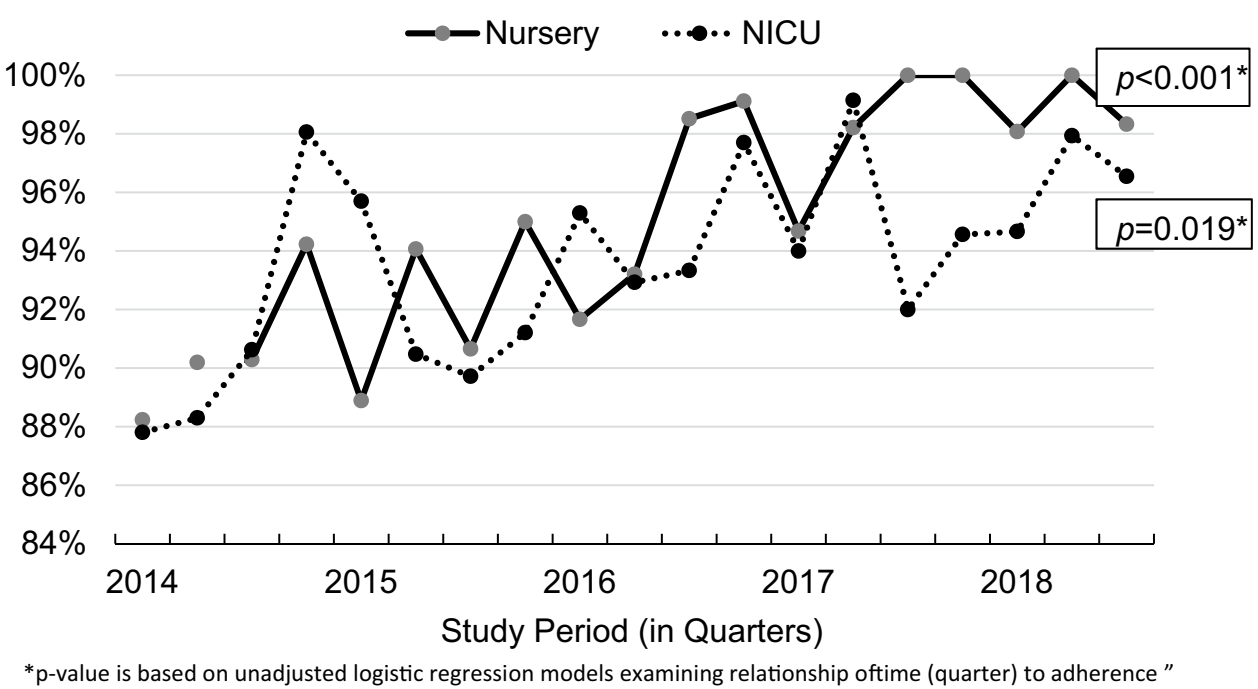


Table 3 Adjusted odds ratio ( $\mathrm{aOR}$ ) of car seat tolerance screening adherence by patient characteristics in NICU and well nursery locations

\begin{tabular}{lll}
\hline & $\begin{array}{l}\text { NICU } \\
\text { aOR }(95 \% \text { CI })\end{array}$ & $\begin{array}{l}\text { Well nursery } \\
\text { aOR }(95 \% \text { CI })\end{array}$ \\
\hline $\begin{array}{l}\text { Hospital type } \\
\text { Academic }\end{array}$ & Reference & Reference \\
Community & $\mathbf{4 . 1 2}(\mathbf{2 . 2 1}, \mathbf{7 . 6 8})^{* * *}$ & $1.20(0.73,1.96)$ \\
Race/ethnicity & & \\
White, non-Hispanic & Reference & Referent \\
Black, non-Hispanic & $0.62(0.37,1.02)$ & $0.72(0.41,1.28)$ \\
Hispanic or Latino & $0.92(0.37,2.26)$ & $0.91(0.34,2.40)$ \\
Asian & $1.49(0.20,11.28)$ & $3.84(0.84,17.59)$ \\
Other/unknown & $1.05(0.36,3.10)$ & $0.77(0.29,2.07)$ \\
Gestational age & $0.84(0.76,0.94)$ & $\mathbf{0 . 4 2}(\mathbf{0 . 3 3}, \mathbf{0 . 5 5})^{* * *}$ \\
Birth weight $(\mathrm{kg})$ & $\mathbf{1 . 8 2}(\mathbf{1 . 0 8}, \mathbf{3 . 1 0})^{* * * *}$ & $1.08(0.64,1.82)$ \\
Female & $0.83(0.54,1.28)$ & $0.93(0.59,1.46)$ \\
Medicaid & $\mathbf{0 . 5 5}(\mathbf{0 . 3 4}, \mathbf{0 . 8 7}) * * *$ & $1.35(0.76,2.40)$ \\
Prefer Spanish & $0.93(0.33,2.66)$ & $0.97(0.29,3.25)$ \\
Discharge year & $\mathbf{1 . 3 0}(\mathbf{1 . 1 1}, \mathbf{1 . 5 2})^{* * * *}$ & $\mathbf{1 . 8 3}(\mathbf{1 . 5 2}, \mathbf{2 . 2 1})^{* * *}$ \\
\hline
\end{tabular}

Bold values represent significance at $\mathrm{p}<0.05$

$* * * p<0.001$

infants with a history of apnea or caffeine exposure had greater adherence (Supplemental Table 2).

\section{Discussion}

While several retrospective studies have described infant predictors of CSTS failure, this is the first to examine clinical factors associated with adherence to institutional CSTS policies. In all hospital units and settings, adherence to CSTS was lowest among term, low birth weight infants compared to preterm infants regardless of weight category. Lower adherence to CSTS policies among term and low birth weight infants is a novel finding. Previous literature suggests that term and low birth weight infants have similar likelihood of CSTS failure as their preterm counterpoints (Bass, 2015; Davis, 2015; McLaurin-Jiang et al., 2019). This is likely due to decreased muscle tone, greater susceptibility to airway obstruction while seated semi-upright, and small size which complicates positioning in car seat.

However, since the AAP's first policy statement on CSTS in 1991, routine screening for T-LBW infants was not specifically mentioned until the 2009 policy update (Bull et al., 2009). The most recent AAP Policy Statement on Safe Transportation of Newborns references low birth weight as $<2.5 \mathrm{~kg}$ which is the cutoff for CSTS used by approximately $50 \%$ of level 2 and 3 nurseries surveyed (Bull et al., 2009; Davis et al., 2013). Comparatively, the criteria used at this institution are more restrictive (cut-off $<2.3 \mathrm{~kg}$ ) which might identify higher-risk infants with higher rates of adherence. As this is the first study to examine CSTS adherence in this manner, we are unable to assess how adherence in this study may generalize to other institutions with more sensitive weight eligibility criteria.

To what extent US hospitals have implemented and routinely conducted CSTS among T-LBW infants is not known. Our findings that T-LBW infants had lower adherence despite explicit inclusion in the CSTS guidelines within this institution is important given that some institutions may not have a written policy for CSTS of term infants. Given that many standard car seats have a weight minimum of 5 lbs $(2.27 \mathrm{~kg})$, beyond detecting significant cardiorespiratory events, routine CSTS for T-LBW infants has the added benefit of ensuring that caregivers are using a weight-appropriate car seat and are able to appropriately position their children in the car seat prior to discharge.

Although the same CSTS policy was in place across nursery and NICU settings, we suspect there are unit level differences in practice that contribute to variance in adherence. The NICU, by design, cares for infants with greater medical complexity than those cared for in the nursery. It is possible that infants in the NICU undergo additional observation or medical management that may influence providers' decisions to complete pre-discharge CSTS. Within the academic nursery setting, the EHR provides prompts/reminders to complete CSTS prior to discharge as it is an element of the admission order set for all preterm infants, which likely improves adherence. However, a similar prompt is not provided for LBW infants, so for this group, CSTS adherence depends more heavily on the medical team to recall that CSTS screening is warranted.

Further analyses supported differences in the acuity of patients cared for at the academic vs. community NICU (e.g., lower gestational age, birth weight, longer length of stay). We excluded patients undergoing tracheostomy or with ventilator dependency to eliminate patients who did not warrant CSTS due to presumed discharge home on continuous monitoring. As a referral center, the academic NICU also transfers some infants back to referring centers which is often done prior to CSTS. Although we accounted for this in our analysis, there may be other unidentified factors unique to the academic setting that were not evaluated in this study. In the community NICU, a history of apnea or caffeine use strongly predicted CSTS adherence.

Although the hospitals in this study are within the same health care system and a 50-mile geographic radius, important sociodemographic differences were noted between the academic and community NICU. The academic NICU cared for a higher percentage of patients with Medicaid (44\% vs 15\%). Unexpectedly, we found a lower rate of CSTS adherence for Medicaid patients in the academic setting which persisted after controlling for relevant factors including race, gestational age, 
birth weight. Furthermore, CSTS adherence among non-Hispanic Black infants was lower than other racial/ethnic groups. While our study lacked the sufficient power to examine racial/ ethnic variation in CSTS, it is possible that racial and socioeconomic characteristics influence implementation of CSTS.

Implicit bias may also impact provider non-adherence. A 2019 systematic review found that racial inequities in NICU care exist at all levels (structure, process, outcome) and correspond with worse patient outcomes (Sigurdson et al., 2019). Indeed racial inequities in breastfeeding rates have been associated with level of adherence to the Baby Friendly Hospital Initiative, an initiative by the World Health Organization and UNICEF to improve support of breastfeeding during newborn hospitalization (Merewood et al., 2019). Because these racial and socioeconomic differences were only seen in one setting at one hospital, it is difficult to generalize these findings or understand the implications. However, the magnitude of differences in adherence within this population warrants prompt attention and evaluation for quality improvement to better understand risks and benefits of CSTS, and subsequently standardize CSTS in this setting.

Non-adherence to institutional clinical guidelines may occur for multiple reasons including provider knowledge, attitude, and behavior; characteristics of the guidelines, health care systems, and implementation strategies (Arts, et al., 2016; Cabana et al., 1999; Gurses et al., 2010); or contraindications or patient preference (Arts et al., 2016). In the case of CSTS, we do not suspect patient preference factors largely into adherence. Only one infant documented parents declining screening during this study period. Alternatively, when comparing premature Black infants to non-Black infants of the same gestational age, the Black infants are known to have improved respiratory outcomes (Jensen et al., 2018; Loftin et al., 2012; MacDorman \& Gregory, 2015; McLaurin-Jiang et al., 2019). In addition, small epidemiologic studies have reported lower risk of CSTS failure among Black infants (Jensen et al., 2018; McLaurin-Jiang et al., 2019). This alternative explanation may lead providers to perceive a decreased risk of car seat associated morbidity for Black compared to non-Black infants and thereby intentionally offer screening less. However, currently recommendations for CSTS do not vary based on infant race and/or ethnicity, and it is important to note that Black infants are at higher risk of dying from SIDS and other sleep related deaths (Mathews \& Macdorman, 2006). A cross-sectional study involving four academic pediatric clinics found that Black, non-Hispanic caregivers had higher odds of non-adherence to car seat safety (AOR $2.4,95 \%$ CI 1.3, 4.4) and sleeping safety (AOR 2.1, 95\% CI $1.3,3.2)$ (Heerman et al. n.d.). The CSTS is potentially an important opportunity to engage parents in specific education related to car seat safety and highlight the risk of improper car seat use on their infant's health. It is unknown whether receipt of CSTS and associated parental education influences child outcomes or parental behaviors with safe use of car seats and infant sleep practices.

Perhaps supported by increasing awareness of populations at risk for failure, the number of CSTS completed annually has increased within this health care system (McLaurin-Jiang et al., 2019). We found increasing adherence to CSTS in both NICU and nursery settings which could be largely contributing to the increasing use of CSTS. With increased screening, there is greater risk for false positive results or unintended harms which have, to our knowledge, not been systematically defined in the setting of CSTS. Additional studies are needed to examine the benefits of CSTS in preterm and T-LBW infants (such as reductions in Brief Resolved Unexplained Events, Sudden Infant Death Syndrome, readmissions, and neurodevelopmental delays).

One limitation of this study is the cross-sectional study design which limits the ability to determine the directionality of relationships between infant factors and adherence. Second, although this study is strengthened by automatic (instead of manual) extraction of EHR data and consistent documentation of CSTS throughout the study period, we lacked information on providers' rationale or decisionmaking that could influence guideline adherence. Third, since guidelines may differ at other institutions, the external validity may be limited. Finally, although demographic and institutional cultural differences exist between the academic and community settings, external validity of this study is enhanced by including two large hospitals with differing socioeconomic demographics and medical acuity.

Although low birth weight term infants have CSTS failure rates similar to preterm infants, adherence to CSTS for these infants is significantly lower in both well-baby nurseries and NICUs. This study further illuminates socioeconomic disparities in adherence to clinical practice guidelines for the most vulnerable infants in the NICU. Such disparities require multicomponent interventions including implicit bias training for clinicians. Our findings also have implications for optimizing and standardizing CSTS processes. Future studies aiming to clarify the benefit of CSTS are needed to ensure appropriate adherence in high-risk populations.

Supplementary Information The online version contains supplementary material available at https://doi.org/10.1007/s10995-021-03220-5.

Author Contributions All authors contributed to the conceptualization of the study and writing and review of the manuscript. SMJ and VR conducted the analyses.

Funding Supported by the National Institute of Diabetes and Digestive and Kidney Diseases of the National Institutes of Health (NIH) under award Number R25DK096944, and the Academic Pediatric Association (APA) RAPID Scholar Program (SMJ); the Health Resources and Services Agency (HRSA T32HP14001-32) (SMJ); the National Center for Advancing Translational Sciences (NCATS), National Institutes of Health, through Grant Award Number UL1TR002489 (all authors). 
The content is solely the responsibility of the authors and does not necessarily represent the official views of the NIH.

Data Transparency De-identified data may be available upon request.

Code Availability Not applicable.

\section{Declarations}

Conflict of interest The authors declare that they have no conflict of interest.

Ethical Approval This study has been approved by the Institutional Review Board and therefore has been performed in accordance with the ethical standards laid down in the 1964 Declaration of Helsinki and its later amendments.

Consent to Participate Not applicable.

Consent to Publish Not applicable.

\section{References}

American Academy of Pediatrics. (1991). Committee on injury and poison prevention and committee on fetus and newborn: Safe transportation of premature infants. Pediatrics, 87, 120-122.

Arts, D. L., Voncken, A. G., Medlock, S., Abu-Hanna, A., \& van Weert, H. C. P. M. (2016). Reasons for intentional guideline nonadherence: A systematic review. International Journal of Medical Informatics, 89, 55-62. https://doi.org/10.1016/j.ijmedinf.2016. 02.009

Bass, J. L. (2015). Car seat-associated hypoxia: Low birth weight term newborns, another group at risk. Pediatrics. https://doi.org/10. 1542/peds.2015-1292

Bull, M. J., Engle, W. A., Smith, G. A., Baum, C. R., Dowd, M. D., Durbin, D. R., Quinlan, K. P., Sege, R. D., Turner, M. S., Weiss, J. C., Wright, J. L., Gilchrist, J., Haverkos, L., Midgett, J. D., Roche, L., Sinclair, A., Warda, L. J., Kozial, B., Stark, A. R., ... Academy of Pediatrics, A. A. (2009). Safe transportation of preterm and low birth weight infants at hospital discharge. Pediatrics, 123(5), 1424-1429. https://doi.org/10.1542/peds.2009-0559

Cabana, M. D., Rand, C. S., Powe, N. R., Wu, A. W., Wilson, M. H., Abboud, P. A. C., \& Rubin, H. R. (1999). Why don't physicians follow clinical practice guidelines?: A framework for improvement. Journal of the American Medical Association. https://doi. org/10.1001/jama.282.15.1458

Davis, N. L. (2015). Car seat screening for low birth weight term neonates. Pediatrics. https://doi.org/10.1542/peds.2014-3527

Davis, N. L., \& Cheo, S. (2020). A national survey of car seat tolerance screening protocols in neonatal intensive care units. Academic Pediatrics. https://doi.org/10.1016/j.acap.2020.02.004

Davis, N. L., Zenchenko, Y., Lever, A., \& Rhein, L. (2013). Car seat safety for preterm neonates: Implementation and testing parameters of the Infant Car Seat Challenge. Academic Pediatrics, 13(3), 272-277. https://doi.org/10.1016/j.acap.2013.01.009

Gurses, A. P., Marsteller, J. A., Ozok, A. A., Xiao, Y., Owens, S., \& Pronovost, P. J. (2010). Using an interdisciplinary approach to identify factors that affect clinicians' compliance with evidencebased guidelines. Critical Care Medicine. https://doi.org/10.1097/ CCM.0b013e3181e69e02

Heerman, W. J., Perrin, E. M., Sanders, L., Shonna Yin, H., CoyneBeasley, T., Bronaugh, A., Barkin SL, \& Rothman, R. (n.d.).
Racial and ethnic differences in injury prevention behaviors among caregivers of infants. https://doi.org/10.1016/j.amepre. 2016.04.020

Huff, K., Rose, R. S., \& Engle, W. A. (2019). Late preterm infants morbidities, mortality, and management recommendations KEYWORDS late preterm morbidity mortality monitoring management KEY POINTS. Pediatric Clinics of North America. https:// doi.org/10.1016/j.pcl.2018.12.008

Jensen, E. A., Foglia, E. E., Dysart, K. C., Aghai, Z. H., Cook, A., Greenspan, J. S., \& DeMauro, S. B. (2018). Car seat tolerance screening in the neonatal intensive care unit: Failure rates, risk factors, and adverse outcomes. Journal of Pediatrics, 194, 60-66. e1. https://doi.org/10.1016/j.jpeds.2017.11.010

Liaw, P., Moon, R. Y., Han, A., \& Colvin, J. D. (2019). Infant deaths in sitting devices. Pediatrics. https://doi.org/10.1542/peds. 2018-2576

Loftin, R., Chen, A., Evans, A., \& Defranco, E. (2012). Racial differences in gestational age-specific neonatal morbidity: Further evidence for different gestational lengths. American Journal of Obstetrics and Gynecology. https://doi.org/10.1016/j.ajog.2011. 12.017

MacDorman, M. F., \& Gregory, E. C. W. (2015). Fetal and perinatal mortality: United States, 2013. National Vital Statistics Reports.

Martin, J. A., Hamilton, B. E., Osterman, M. J. K., Driscoll, A. K., \& Drake, P. (2018). Births: Final data for 2017. National Vital Statistics Reports: From the Centers for Disease Control and Prevention, National Center for Health Statistics, National Vital Statistics System.

Mathews, T. J., \& Macdorman, M. F. (2006). Infant mortality statistics from the 2006 period linked birth/infant death data set. In National vital statistics reports (Vol. 58).

McLaurin-Jiang, S., Hannon, E. A., Seashore, C., Orr, C. J., Ritter, V., $\&$ Flower, K. B. (2019). Recent trends in infant car seat tolerance screening failure within a large health care system, 2014-2018. Hospital Pediatrics, 9(10), 813-817. https://doi.org/10.1542/ hpeds.2019-0078

Merewood, A., Bugg, K., Burnham, L., Krane, K., Nickel, N., Broom, S., Edwards, R., \& Feldman-Winter, L. (2019). Addressing racial inequities in breastfeeding in the southern United States. Pediatrics, 143(2), e20181897. https://doi.org/10.1542/peds.2018-1897

Pilley, E., \& McGuire, W. (2006). Pre-discharge "car seat challenge" for preventing morbidity and mortality in preterm infants. Cochrane Database of Systematic Reviews. https://doi.org/10. 1002/14651858.CD005386.pub2

Schutzman, D. L., Salvador, A., Janeczko, M., Weisberg, L., Tran, N., \& Porat, R. (2013). A comparison of the infant car seat challenge and the polysomnogram at the time of hospital discharge. Archives of Disease in Childhood: Fetal and Neonatal Edition. https://doi. org/10.1136/archdischild-2012-303244

Sigurdson, K., Mitchell, B., Liu, J., Morton, C., Gould, J. B., Lee, H. C., Capdarest-Arest, N., \& Profit, J. (2019). Racial/ethnic disparities in neonatal intensive care: A systematic review. Pediatrics. https://doi.org/10.1542/peds.2018-3114

Willett, L. D., Leuschen, M. P., Nelson, L. S., \& Nelson, R. M. (1986). Risk of hypoventilation in premature infants in car seats. The Journal of Pediatrics. https://doi.org/10.1016/S0022-3476(86) 80379-1

Williams, L. E., \& Martin, J. E. (2003). Car seat challenges: Where are we in implementation of these programs? Journal of Perinatal and Neonatal Nursing. https://doi.org/10.1097/00005237-20030 4000-00008

Publisher's Note Springer Nature remains neutral with regard to jurisdictional claims in published maps and institutional affiliations. 